\title{
Recalcitrant Macular Hole Closure by Autologous Retinal Transplant Using the Peripheral Retina
}

This article was published in the following Dove Press journal: Clinical Ophthalmology

\author{
Keiko Yamada ${ }^{1,2}$ \\ Takatoshi Maeno ${ }^{3}$ \\ Shunji Kusaka (D) ${ }^{4}$ \\ Jorge G Arroyo ${ }^{2}$ \\ Mitsunori Yamada ${ }^{5}$ \\ 'Department of Ophthalmology, Faculty \\ of Medicine, Osaka University, Osaka, \\ Japan; ${ }^{2}$ Department of Ophthalmology, \\ Harvard University Beth Israel \\ Deaconess Medical Center, Boston, MA, \\ USA; ${ }^{3}$ Department of Ophthalmology, \\ Toho University Sakura Medical Center, \\ Chiba, Japan; ${ }^{4}$ Department of \\ Ophthalmology, Faculty of Medicine, \\ Kindai University, Osaka, Japan; ${ }^{5}$ Yamada \\ Eye Clinic, Tokushima, Japan
}

Video abstract

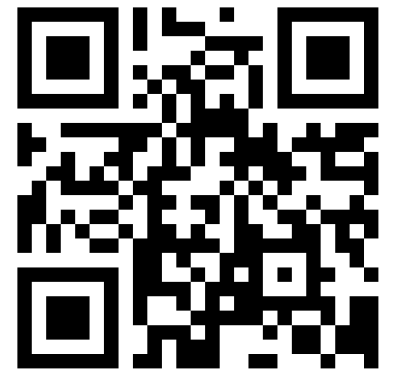

Point your SmartPhone at the code above. If you have a $Q R$ code reader the video abstract will appear. Or use: https://youtu.be/RzbBo-xxq0g

Correspondence: Keiko Yamada Email meyamada@mb3.tcn.ne.jp
Purpose: The peripheral adult human retina has been found to contain neuroepithelial stem cells. In this study, we examined the efficacy of an auto-transplant of peripheral retina into refractory macular holes $(\mathrm{MH})$ from both anatomic and physiologic perspectives.

Methods: The population consisted of four female patients aged 72, 82, 65 and 84 years (cases 1-4, respectively) with persistent refractory MH status; internal limiting membrane (ILM) peeling (case 1), ILM transplant (case 2), and inverted ILM (cases 3 and 4 with myopic $\mathrm{MH}$ ). In all our cases, retinal grafts were harvested beyond the equator from the far retinal periphery using curved horizontal scissors and gently moved toward the $\mathrm{MH}$ using a forceps. A 25-G manipulator with a silicone ball tip was used to tuck the trimmed graft into the $\mathrm{MH}$, followed by fluid-air exchange and infusion of silicone oil, which was removed three months later.

Results: Partial restoration and integration of the outer retinal layer were confirmed on an OCT-B scan imaging. The visual acuity (VA) was improved in all cases: 1.2 to $1.0 \log$ MAR (case 1), 2.0 to $1.3 \operatorname{logMAR}$ (case 2), 2.3 to $1.4 \log$ MAR (case 3 ) and 2.0 to $1.0 \log$ MAR (case 4). Microperimetry showed improved retinal sensitivity in every case. No intra- or post-operative complications were observed.

Conclusion: Under pathological conditions, the Müller glia reportedly serves as a source of neuronal progenitor cells in regenerating retina, continuing to divide and migrate to the outer nuclear layer thus replacing lost photo-receptors. Although the histological findings remain unknown, the positive anatomic and physiologic outcomes of the auto-transplanted retinal flap in our series suggest that this technique may offer an effective option for treating recalcitrant MH. Further studies are warranted.

Keywords: retina, macular hole, vitrectomy, stem cell, transplant; Müller glia

\section{Introduction}

Since the original surgical rationale to fix macular holes $(\mathrm{MH})$ was described by Kelly and Wendel in 1991, methods to repair this pathology have been continuously refined. In 1997, Eckardt et $\mathrm{al}^{3}$ reported that internal limiting membrane (ILM) removal is effective for preventing the recurrence of $\mathrm{MH}$. Michalewska et $\mathrm{al}^{4}$ then expanded the literature by introducing an inverted ILM flap technique that can decrease the ratio of post-operative flat-open or flat-closure $\mathrm{MH}$ based on the presence or absence of bare retinal pigment epithelium. After this, Morizone, Shiraga et $\mathrm{al}^{5}$ demonstrated the effectiveness of the free ILM flap transplantation, even if the ILM was removed in previous procedures. These inverted or transplanted ILM flaps are believed to work as a scaffold that encourage Müller cell gliosis resulting in $\mathrm{MH}$ closure. Grewal, Mahmoud et $\mathrm{al}^{6,7}$ proposed an autologous 
retinal transplantation (ART) for refractory $\mathrm{MH}$ closure and suggested that transplanted Müller cell progenitors may be able to replace lost photo-receptors. ${ }^{1,2}$

The purpose of this case study is to describe the anatomic and physiologic outcomes of ART for recalcitrant $\mathrm{MH}$ closure in four cases treated using our own techniques.

\section{Methods}

In the present study, we report the cases of four pseudophakic patients who underwent ART surgery after previously receiving several vitrectomy procedures, including ILM peeling for $\mathrm{MH}$. This retrospective observational study was conducted at the Yamada Eye Clinic in Tokushima, Japan from September 9, 2017 (case 1) to September 22, 2018 (case 4). All patients were followed for at least 6 months after surgery. The institutional review board approved the current study, and written informed consent was obtained from all of the participants in accordance with the tenets of the Declaration of Helsinki.

Vitrectomy surgery was performed using a $23-G$ dual cutter $(8000 \mathrm{rpm})$ of an EVA surgical system (DORC, Zuidland, the Netherlands) under prism contact lenses (HHV;HOYA surgical optics, Osaka, JAPAN) and a wideangle viewing system (Central Retina and HRX; Volk Optical Inc. USA). Triamcinolone acetonide was injected to visualize the vitreous gel and areas of vitreoretinal adhesion. Endoillumination with gentle scleral indentation helped to identify any pathological features in the peripheral retina. Brilliant Blue $\mathrm{G}$ was used to stain around the MH within the arcade in order to confirm the extent and adequacy of the previous ILM peel. A neurosensory retinal harvest site was selected in the superior temporal peripheral retina, beyond the arcade. The size of the harvested tissue was set to twice the size of the $\mathrm{MH}$, and then endolaser barricade was performed in a circular manner. The ART graft was made using the 23-G curved horizontal intra-ocular micro scissors (SC 23. R03, Vitreq, the Netherlands); the graft was not completely released, as a hinge was left in place.

The flattened MH edge was lifted up using a soft-tipped flute needle (25-G-Brush Backflush, BF25.D05, Vitreq) to make some space underneath the edge. Then, viscoelastic material (Viscoat, Alcon Japan Ltd.) was put into the MH. After endo-diathermy of the blood vessels at the edge of the harvest site, the ART tissue was cut from the residual hinge and carried toward the MH using a 23-G micro forceps while maintaining correct tissue orientation (Au-2286, K06;
DORC) (Figure 1A and B). The trimmed free ART flap was positioned on the Viscoat-covered $\mathrm{MH}$. The graft was gently nestled into the $\mathrm{MH}$ and was buried under the elevated edge of the MH using a 25-G Maeno-Awh super manipulator (Katalyst Surgical, LLC, MO, USA) (Figure 1C and D). ${ }^{8,9}$

The iatrogenic peripheral retinal hole caused by harvesting the tissue was then sealed with additional endolaser photocoagulation. Fluid air exchange (FAX) was slowly repeated using a soft-tipped flute needle, changing the position between the $\mathrm{MH}$ lesion and optic disc before the air was replaced with 1000 centistoke silicone oil (SO). Under SO, the ART flap was then confirmed to be centered in the MH. All patients were instructed to keep face-down positioning for two days after surgery as much as possible.

The preoperative and postoperative examinations included indirect slit lump ophthalmoscopy (75SL; Zeiss, Oberkochen. Germany) with high power convex lenses (Volk Optical, Inc., Mentor, OH, USA) and OCT imaging using Cirrus spectral-domain OCT and Elite swept-source OCT (Carl Zeiss Meditec, Dublin, CA, USA) for the morphological diagnosis of MH.

The best corrected visual acuity (BCVA) of each patient was assessed using a back-illuminated ETDRS chart in six months follow-up. The measured BCVA was presented as the logarithm of the minimum angle of resolution (LogMAR). Functional changes in this study were confirmed by BCVA and MP-3 microperimetry (Nidek, Aichi, Japan) as the mean retinal sensitivity.

\section{Results}

Four female patients (mean age, 75.8 \pm 7.7 years; range, 66-81 years) had refractory MHs after failed surgeries. Case 1 had previously undergone ILM peeling, case 2 had undergone vitrectomy twice (the first procedure was combined with ILM peeling, while the second procedure was combined with free ILM transplantation) and cases 3 and 4 had undergone inverted ILM transplantation. All 4 patients had MHs larger than $400 \mu \mathrm{m}$, including 2 highmyopic MHs with posterior staphyloma (axial length: $33.75 \mathrm{~mm}$ [case 3] and $29.93 \mathrm{~mm}$ [case 4], in comparison to $24.04 \mathrm{~mm}$ [case 1] and $23.70 \mathrm{~mm}$ [case 2]).

No intraoperative or postoperative complications were observed in this case series. Free ART flaps were retained in MHs and their closure was eventually achieved in all cases as confirmed by OCT imaging after surgery. The MHs with the inserted ART flap and their retinal layers were delineated. The white arrows in Figure 2 indicate the fovea. Integration of the ART graft with donor tissue (Figure 2-1B*) and 

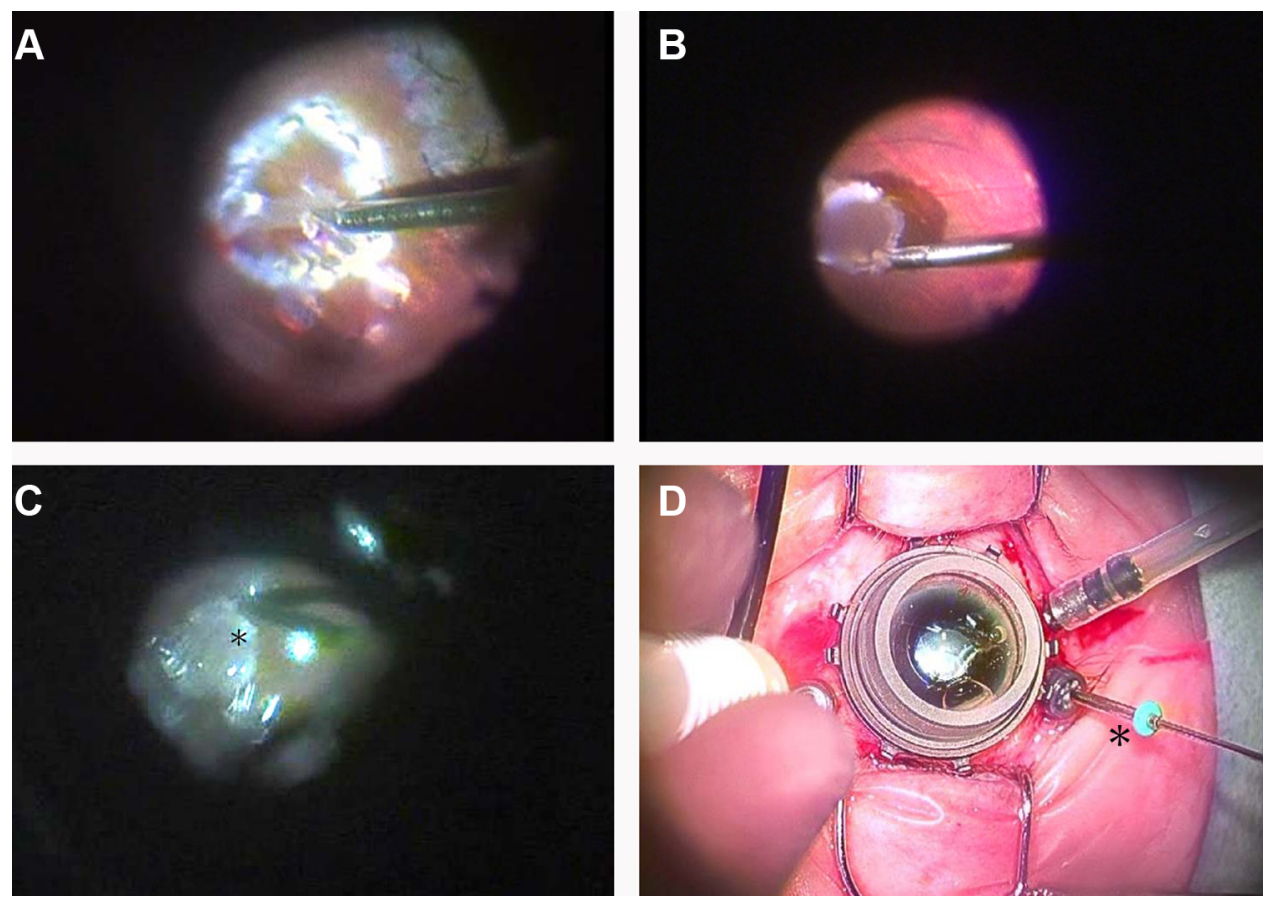

Figure I The surgical video capture shots of the autologous retinal transplant in this study (casel). (A) A neurosensory retina harvest site was selected superior to the temporal arcade. Endolaser barricade was applied in a circular manner followed by endo-diathermy to blood vessels at the site edges. The edge of the graft was cut using 23-G curved horizontal scissors with part of the graft still attached. (B) A full-thickness neurosensory retinal free flap was released from the residual hinge and gently moved toward the MH using 23-G micro forceps. (C) A free-ART flap was inserted into the floating edge of the MH using the Maeno-Awh manipulator* under viscoelastic devise and the air. (D) The manipulator had a $2 \mathrm{~mm}$ silicone tip* at the end of a movable $25-\mathrm{G}$ stainless steel shaft to facilitate smooth insertion into the trocar with the closure bulb. ${ }^{9}$

Abbreviations: ART, autologous retinal transplant; $\mathrm{MH}$, macular hole.

intraretinal cystoid change in the graft (Figure 2-2B**) were suspected based on OCT images of the fovea.

Regarding respect to the functional outcomes, the BCVA improved in all cases at the final follow-up examinations (1.2 to 1.0 [case 1], 2.0 to 1.3 [case 2], 2.3 to 1.4 [case 3] and 2.0 to 1.0 [case 4]). The mean BCVA was 1.88 \pm 0.47 (range $1.2-2.3$ ) before surgery and $1.17 \pm 0.21$ (range 1.0-1.4) at six months after surgery. However, there was no post-operative improvement in the BCVA of all cases in the first six months. The MP-3 findings of each patient are shown in Figure 2 (1-c, 2-c, 3-c and 4-c). A custom grid was designed to assess the macular region sensitivity, consisting of 33 points located at $0^{\circ}, 2^{\circ}, 4^{\circ}, 6^{\circ}, 8^{\circ}, 10^{\circ}$ and $12^{\circ}$ from the fixation site. Every case retained some retinal sensitivity points at the final visit.

\section{Discussion}

Recent surgical techniques are said to have improved the success rate of MH surgery to $80 \%-95 \%$. Nevertheless, compared with idiopathic MHs, the postoperative closure rate and functional outcomes for larger MHs, high-myopic MHs and long-standing MHs including recurrent and secondary MHs are significantly lower, and some cases require repeated surgery. ${ }^{10,11}$ Moreover, repeated operations may be less successful in cases where the initial surgery successfully relieved all vitreous traction through an ILM peel.

While Mahmoud and colleagues proposed ART as a method for the closure of refractory myopic MHs, this promising technique deserves further discussion. ${ }^{6,7,10}$ The key points of our method are as follows: First, the initial harvest graft size should be about twice as large as the $\mathrm{MH}$, considering the need for trimming and potential shrinkage over time. Second, the transplanted neurosensory retina is thicker than the ILM, and the neurosensory retinal flap does not easily drift away during FAX. Third, the graft should be positioned over the base of the MH and inserted under the hole edges using a manipulator and viscoelastic material.

In the present study, all patients experienced an improvement in VA associated with some anatomic integration of ART. Whether or not ART is a worthwhile intervention in chronic MH patients with poor baseline visions remains controversial. ${ }^{7}$ Anatomic success is certainly a prerequisite for visual success, but evaluating visual success can be difficult. Improvements in VA are notoriously difficult to measure when the range of VA is 

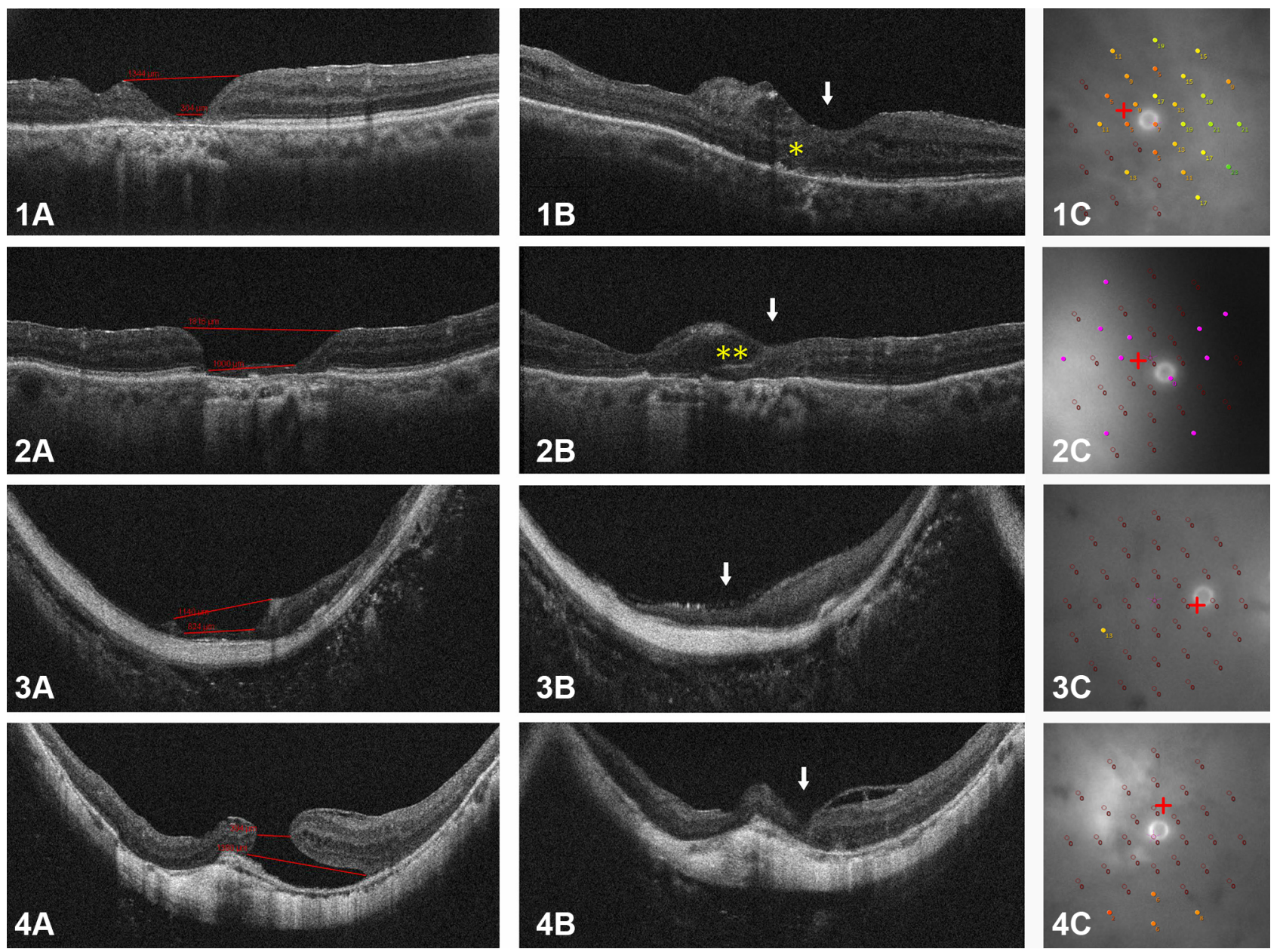

Figure 2 The results of the autologous retinal transplant in this study. Case I: A 72-year-old woman (OD) experienced failed vitrectomy with the ILM removal. Case 2: An 82-year-old woman (OS) experienced failed vitrectomy with free ILM transplantation following ILM removal. Case 3: A 65-year-old woman (OD) experienced failed vitrectomy with an inverted ILM flap technique. Case 4: An 84-year-old woman (OD) experienced failed vitrectomy with an inverted ILM flap technique. I-4(A): preoperative OCT images showing the unclosed $\mathrm{MH}$. I-4(B): postoperative OCT images after ART showing the closed MH (white arrows). Yellow * in top panel of IB: suspected regenerating outer retinal layers. Yellow $* *$ in second row panel $2 \mathrm{~B}$ : cystoid change in the graft. $\mathrm{I}-4(\mathrm{C})$ : Microperimetry in $12^{\circ}$ fovea area showing retinal sensitivity of fixation view point at final visit. The microperimetry results are shown for differential threshold locations ranging from 0 to $34 \mathrm{~dB}$.

Abbreviations: ART, autologous retinal transplant; MH, macular hole; ILM, internal limiting membrane; OD, right eye; OS, left eye; OCT, optical coherence tomography.

poor, which makes the evaluation of the consequential functional benefit even more difficult. The visual outcome seems to be strongly correlated with factors such as the pre-operative VA, MH diameter, and the interval from the previous intervention.

As shown in Figure 2-1B and 2B, partial restoration of the outer retinal layers and intraretinal cystoid change in the graft may indicate the integration of the graft with the surrounding retinal tissues, and are consistent with previous studies. ${ }^{6,7}$ Some studies have suggested that the postoperative recovery of the outer retinal structure is the decisive factor affecting the postoperative vision. Recovery of the ellipsoid zone (EZ) and the external limiting membrane (ELM) have been reported to contribute to improvement in the VA in MH cases after surgery. ${ }^{11}$
There are several therapeutic methods that transfer the retina cells differentiated from iPS cells or ES cells to the macula. Since the peripheral retina is autologously transplanted into the central retina, leading to desirable outcomes from anatomic and functional viewpoints, it could indicate a paradigm shift in the area of retinal regeneration medicine. It doesn't seemthat a retinal free flap could transmit a signal to the optic nerve, as the retinal circulation is interrupted. Figure 3 shows that, under pathological conditions, the Müller glia serve as a source of neuronal progenitor cells (indicated in green) in the regenerating retina. The cells continue to divide and use the cell processes to migrate to the outer nuclear layer, thereby replacing lost photo-receptors. It is hypothesized that the transplanted retinal flap may represent a source of stem cells that can migrate into the native retina and 

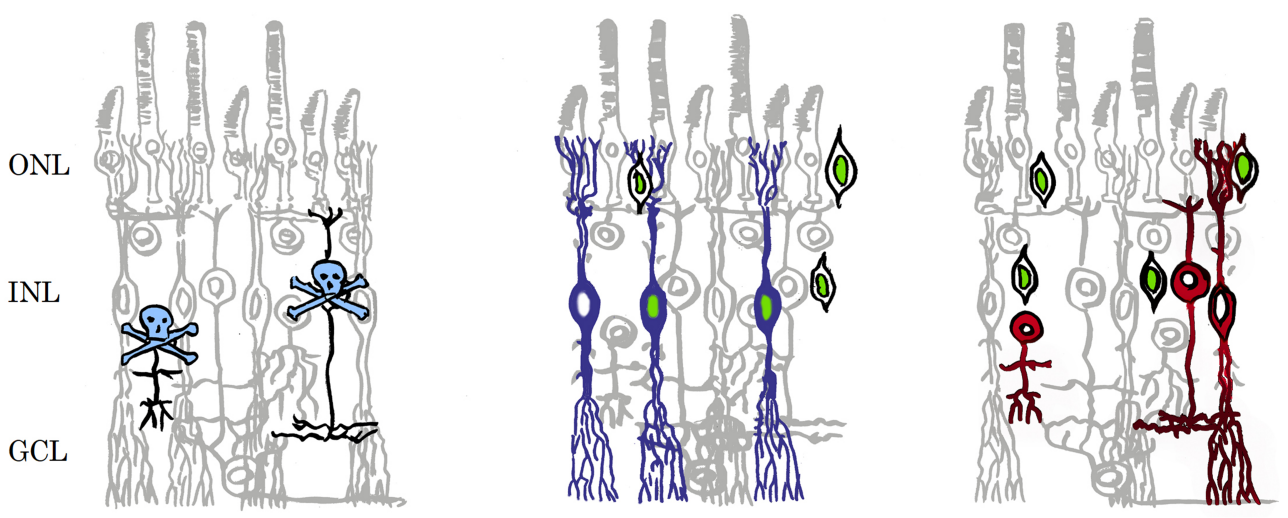

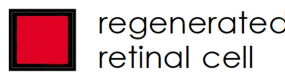

Pax6 and Chx10

BrdU-labeled cell

neurofilament

cell death

Figure 3 Schematic diagram illustrating the response of Müller cells to NMDA-induced damage. Müller cells can act as neutral progenitor cells in the chicken retina. The progenitor cells migrate into the ONL where they continue to proliferate and generate photo receptor cells. Modified from Fischer and Reh (2003).

Abbreviations: ONL, outer nuclear layer; INL, inner nuclear layer; GCL, ganglion cell layer; NMDA, N-methyl-d-aspartate; Pax6 and ChxI0, homeodomain transcription factors (Fischer and Reh, 200I); BrdU, bromodeoxyuridine. ${ }^{2}$

promote the repair of the outer retinal layers. However, if it is true that Müller cells that retain retinal progenitor properties in adults become increasingly confined to the periphery, it would make sense to harvest a retinal flap from this area. ${ }^{1,2}$ This would be potentially useful in a wide spectrum of retinal pathologies, including but not limited to, central artery occlusions and retinal detachment. ${ }^{12}$

The main limitation of this study is the small sample size and short follow-up period. However, our case report and discussion of the key aspects of the procedure offer intriguing results. In conclusion, we find that vitrectomy combined with an ART graft from the peripheral retina may be an effective surgical option for large refractory MHs after failed surgery with ILM removal or transplantation.

\section{Acknowledgment}

The authors would like to express many thanks to Mr. Brendan Seto in Beth Israel Deaconess Medical Center for his kind support.

\section{Disclosure}

The authors report no conflicts of interest in this work.

\section{References}

1. Erik OJ, Rebecca CF, Reka A, et al. Activation of neural progenitor cells in human eyes with proliferative vitreoretinopathy. Exp Eye Res. 2012;98:28-36. doi:10.1016/j.exer.2012.03.008
2. Reichenbach A, Bringmann A. New functions of Müller cells. Glia. 2013;61(5):651-678. doi:10.1002/glia.22477

3. Eckardt C, Eckardt U, Groos S, et al. Removal of the internal limiting membrane in macular holes. Clinical and morphological findings. Ophthalmologe. 1997;94(8):545-551. doi:10.1007/s003470050156

4. Michalewska Z, Michaleaski J, Dulczewska-Cichecka K, et al. Inverted internal limiting membrane flap technique for surgical repair of myopic macular holes. Retina. 2014;34(4):664-669. doi:10.1097/ IAE.0000000000000042

5. Morizane Y, Shiraga F, Kimura S, et al. Autologous trans plantation of the internal limiting membrane for refractory macular holes. $\mathrm{Am}$ J Ophthalmol. 2014;157(4):861-869. doi:10.1016/j.ajo.2013.12.028

6. Grewal DS, Mahmoud TH. Autologous neurosensory retinal free flap for closure of refractory myopic macular holes. JAMA Ophthalmol. 2016;134(2):229-230. doi:10.1001/jamaophthalmol.2015.5237

7. Grewal DS, Charles S, Parolini B, et al. Autologous retinal transplant for refractory macular holes; Multicentor International Collaborative Study Group. Ophthalmology. 2019;126(10):1399-1408. doi:10.10 16/j.ophtha.2019.01.027

8. Kumar A, Tinwala SI, Gogia V, et al. Tapping of macular hole edges: the outcomes of a novel technique for large macular holes. Asia Pac $J$ Ophthalmol. 2013;2(5):305-309. doi:10.1097/APO.0b013e3182 9a1919

9. Maeno T, Nagaoka T, Ubuka M, et al. New surgical instrument for autologous internal limiting membrane transplantation for the treatment of refractory macular holes. Retina. 2018;38(3):643-645. doi:10.1097/IAE.0000000000001890

10. Ding C, Li S, Zeng J. Autologous neurosensory retinal transplantation for unclosed and large macular holes. Ophthalmic Res. 2019;61 (2):88-93. doi:10.1159/000487952

11. Wakabayashi T, Fujiwara M, Sakaguchi H, et al. Microstructure and visual acuity in surgically closed macular holes: spectral-domain optical coherence tomographic analysis. Ophthalmology. 2010;117 (9):1815-1824. doi:10.1016/j.ophtha.2010.01.017

12. Hayreh SS, Zimmerman MB, Kimura A, et al. Central retinal artery occlusion: retinal survival time. Exp Eye Res. 2004;78(3):723-736. doi:10.1016/S0014-4835(03)00214-8 


\section{Publish your work in this journal}

Clinical Ophthalmology is an international, peer-reviewed journal covering all subspecialties within ophthalmology. Key topics include: Optometry; Visual science; Pharmacology and drug therapy in eye diseases; Basic Sciences; Primary and Secondary eye care; Patient Safety and Quality of Care Improvements. This journal is indexed on PubMed
Central and CAS, and is the official journal of The Society of Clinical Ophthalmology (SCO). The manuscript management system is completely online and includes a very quick and fair peer-review system, which is all easy to use. Visit http://www.dovepress.com/ testimonials.php to read real quotes from published authors. 